\title{
RT-qPCR Methods to Support Pharmacokinetics and Drug Mechanism of Action to Advance Development of RNAi Therapeutics
}

\author{
Elena Castellanos-Rizaldos, ${ }^{\star}$ Christopher R. Brown, ${ }^{\star}$ Sean Dennin, Joohwan Kim, Swati Gupta, \\ Diana Najarian, Yongli Gu, Krishna Aluri, Jennifer Enders, Kirk Brown, and Yuanxin Xu
}

\begin{abstract}
The goal of this study was to develop a reverse transcription quantitative polymerase chain reaction (RT-qPCR) method for the accurate quantification of chemically modified small interfering RNA (siRNA) including but not restricted to thermally destabilizing modifications such as glycol nucleic acid (GNA). RT-qPCR was found to be superior to mass spectrometry-based siRNA detection in terms of sensitivity and throughput. However, mass spectrometry is still the preferred method when specific metabolite detection is required and is also insensitive to siRNA chemical modifications such as GNA. The RT-qPCR approach can be optimized to take chemical modifications into account and works robustly in different matrices without optimization, unlike mass spectrometry. RT-qPCR and mass spectrometry both have their strengths and weaknesses for the detection of siRNA and must be used appropriately depending on the questions at hand. Considerations such as desired throughput, assay sensitivity, and metabolite identification must be weighed when choosing which methodology to apply.
\end{abstract}

Keywords: RT-qPCR, small interfering RNA, pharmacokinetics, pharmacodynamics

\section{Introduction}

$\mathbf{T}$ HE FIELD OF RNA therapeutics that uses a new class of therapeutics, small interfering RNAs (siRNAs), has advanced rapidly in the past few years due to the recent approvals of ONPATTRO ${ }^{\circledR}$ and GIVLAARI ${ }^{\circledR}$ by the U.S. Food and Drug Administration (FDA) in August 2018 and November 2019, respectively [1,2].

siRNAs trigger the RNA interference (RNAi) pathway, an intrinsic mechanism of post-transcriptional gene silencing in eukaryotic cells (Fig. 1) [3]. During this process, endogenous short double-stranded RNAs [ $\sim 21$ base pairs (bp) with 2-base staggered $3^{\prime}$ ends] associate with multiple protein components in the cytoplasm to form the RNA-induced silencing complex (RISC) [3]. Once loaded, the passenger (sense) strand is cleaved, unwound, removed from the complex, and degraded by cytoplasmic nucleases. The RISC-loaded guide (antisense) strand then binds through Watson-Crick base-pairing to a target complementary messenger RNA (mRNA). Subsequently, this process triggers the endonucleolytic cleavage of the complementary target mRNA on the site opposite nucleotides $10-11$ of the antisense strand of the siRNA $\left(5^{\prime}-3^{\prime}\right.$ orientation) [4,5]. RNAi can also be induced by exogenous siRNA duplexes, administered either subcutaneously (SC) or intravenously. After entering the cytoplasm of the cell, synthetic siRNAs are loaded into RISC and effectively trigger endonucleolytic cleavage of target mRNA.

To achieve targeted delivery to hepatocytes without formulating siRNAs into lipid nanoparticles, a new generation of siRNA-conjugates was developed. siRNAs conjugated to a triantennary $N$-acetylgalactosamine (GalNAc) are recognized with high affinity by the asialoglycoprotein receptor, which is almost exclusively expressed in hepatocytes. After endocytosis, siRNAs proceed through the intracellular trafficking pathway and are released into the cytoplasm by an asyet undefined mechanism. Once in the cytoplasm, siRNAs suppress the production of target protein through cleavage of mRNA as described earlier. Focused receptor-mediated uptake significantly improves tissue specificity and enhances the therapeutic index of siRNA, resulting in more efficacious treatments with less frequent dosing [6-8].

To better understand the absorption, distribution, metabolism, and excretion (ADME) properties of GalNAc-siRNA, there is an increasing need to adapt current bioanalytical tools to achieve the necessary sensitivity to provide accurate quantification from various matrices, including biofluids 


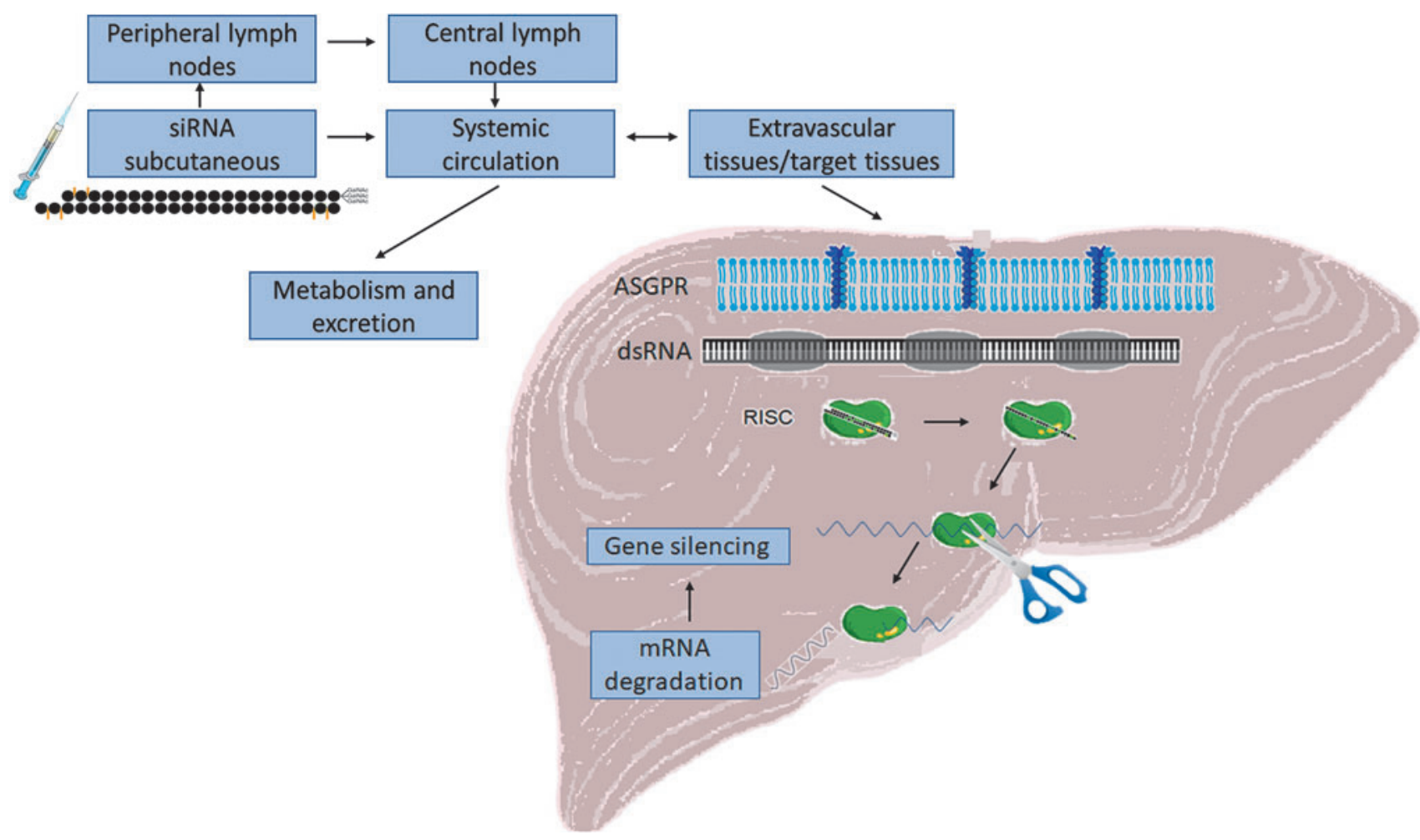

FIG. 1. Uptake of GalNAc-conjugated siRNA by hepatocyte ASGPR mediate oligonucleotide delivery from the injection site to the target tissue (liver) to induce targeted gene silencing through the intrinsic RNAi pathway. ASGPR, asialoglycoprotein receptors; GalNAc, $\mathrm{N}$-acetylgalactosamine; RNAi, RNA interference; siRNA, small interfering RNA.

such as plasma or serum. To achieve this, traditional bioanalytical methods such as mass spectrometry, hybridizationbased high-performance liquid chromatography-fluorescence assay, and probe hybridization assays have proven to be very useful tools to advance the development of these new therapies [9]. Mass spectrometry is still the only method that can accurately quantify and discriminate metabolites from the full-length siRNA (Table 1) with a resolution of $1 \mathrm{bp}$ [10-14]. These methods provide sensitivity only in the $\mathrm{ng} / \mathrm{mL}$ range, whereas tools such as reverse transcription quantitative polymerase chain reaction (RT-qPCR) can reach sensitivities up to $1,000,000$ times greater $(\mathrm{pg} / \mathrm{mL}$ to $\mathrm{fg} / \mathrm{mL})$. In addition, assay development and hands-on time for sample analysis are longer for both mass spectrometry and hybridization approaches compared with RT-qPCR.
Modifications to siRNAs such as $2^{\prime}-O$-methyl (2'-OMe), $2^{\prime}$-deoxy-2'-fluoro $\left(2^{\prime}-\mathrm{F}\right)$, and phosphorothioate (PS) linkages have been widely used to stabilize siRNAs, reduce immune stimulation, and enhance siRNA potency [15]. More recently, thermally destabilizing modifications such as glycol nucleic acid (GNA) have been incorporated to mitigate offtarget activity of siRNAs [16]. Other modifications commonly found in siRNAs include $3^{\prime}$ - $O$-methyl- $2^{\prime}$-phosphate (3'-OMe) and unlocked nucleic acid.

The increasing need for sensitive detection to assess RISC loading, the presence of metabolites with pharmacodynamic activity, and the siRNA length $(<23 \mathrm{bp})$ have presented new challenges associated with siRNA quantification. In addition, although guidelines describing the minimum information necessary for evaluation of qPCR experiments have been

Table 1. Comparison of Methods Used for Small Interfering RNA Quantification

\begin{tabular}{|c|c|c|c|c|c|}
\hline $\begin{array}{l}\text { Method } \\
\text { of quantification }\end{array}$ & $L L O Q^{\mathrm{a}}$ & $\begin{array}{c}\text { Metabolite detection } \\
\text { (in addition to full-length) }\end{array}$ & Throughput & $\begin{array}{l}\text { One assay per } \\
\text { siRNA sequence }\end{array}$ & $\begin{array}{l}\text { Tissue matrix } \\
\text { interference }\end{array}$ \\
\hline RT-qPCR & $\approx \mathrm{pg}-\mathrm{fg} / \mathrm{mL}$ & $\begin{array}{l}\text { Can be tuned to detect } \\
\text { individual metabolites }\end{array}$ & $\begin{array}{l}\text { High }(384 \text {-well } \\
\text { plate) }\end{array}$ & Yes & No \\
\hline Hybridization & $\approx 1 \mathrm{ng} / \mathrm{mL}$ & Captures all metabolites & $\begin{array}{l}\text { Mid/high (96-well } \\
\text { plate) }\end{array}$ & Yes & Yes \\
\hline Mass spectrometry & $\approx 5-10 \mathrm{ng} / \mathrm{mL}$ & $\begin{array}{l}\text { Detects full-length and } \\
\mathrm{N}-1 \text { metabolite } \\
\text { simultaneously }\end{array}$ & Mid & No & Yes \\
\hline
\end{tabular}

${ }^{a}$ LLOQ. May vary for different compounds and matrices.

${ }^{b}$ Assay can be used for siRNAs that share the same nucleotide sequence despite having different chemical modifications.

LLOQ, lower limit of quantification; RT-qPCR, reverse transcription quantitative polymerase chain reaction; siRNA, small interfering RNA. 
proposed by academic laboratories [17], there is still a lack of regulatory guidance and industry practice describing assay development and validation. This article presents our current RT-qPCR assay that was developed for the quantification of chemically modified siRNA, including siRNAs with thermally destabilizing modifications such as GNA.

\section{Materials and Methods}

\section{Small interfering RNAs}

The eight siRNA molecules used in this study were synthesized by Alnylam Pharmaceuticals, Inc. (Cambridge, MA) as described by Nair et al. [7]. The identities and purities of all oligonucleotides were confirmed by electrospray ionization mass spectroscopy and ion exchange high-performance liquid chromatography, respectively. Although the siRNA molecules differ in sequence, all contain phosphorothioate bonds as well as 2'-OMe and 2'-F modifications and all are conjugated to a triantennary GalNAc moiety. The siRNA stock solutions were prepared at $500 \mathrm{nM}$ in PBST (phosphatebuffered saline, PBS, from $10 \times$ PBS; ThermoFisher Scientific, Waltham, MA) with $0.25 \%$ Triton X-100 (Sigma-Aldrich, St. Louis, MO) and stored frozen at $-20^{\circ} \mathrm{C}$.

\section{Preparation of standard curves and quality control samples}

On the day of analysis, standards were prepared by performing fivefold serial dilutions in PBST of a $500 \mathrm{nM}$ siRNA stock solution using the epMotion ${ }^{\circledR} 5075 \mathrm{t}$ liquid handler (Eppendorf North America, Hauppauge, NY) and the epMotion Editor software (version 40.6). Standards were kept on ice until RT-qPCR analysis. Quality control (QC) samples were also prepared in PBST at 50, 5, 0.5, 0.05 , and $0.005 \mathrm{nM}$. Concentrations in $\mathrm{nM}$ were then converted to $\mathrm{ng} / \mathrm{mL}$ using the corresponding siRNAs molecular weight.

Two nontemplate controls are included in all experiments. The first contains the water used to prepare the transcription master mix and the second contains the PBST buffer used as diluent for samples and standards.

\section{Preparation of plasma, serum, and tissue samples for total siRNA quantification}

Serum and plasma samples were diluted in PBST $(0.25 \%$ Triton X-100) using a minimum 100-fold dilution to avoid matrix interference and protein precipitation during the heating step.

Approximately $50 \mathrm{mg}$ of liver tissue from treated and untreated animals was processed to powder as described by Foster et al. [15] and stored at $-80^{\circ} \mathrm{C}$. All in vivo experiments were performed according to the Institutional Animal Care and Use Committee (IACUC)'s regulations at Alnylam Pharmaceuticals, Inc. These protocols followed the U.S. Government Principles for the Utilization and Care of Vertebrate Animals Used in Testing, Research, and Training.

\section{Reverse transcription preparation of $c D N A$}

To determine tissue siRNA concentrations, $\sim 10 \mathrm{mg}$ of powdered tissue was resuspended to a final concentration of $10 \mathrm{mg} / \mathrm{mL}$ in PBST. Diluted samples were incubated on a dry block (VWR ${ }^{\circledR}$ Advanced dry block heater; VWR) at $95^{\circ} \mathrm{C}$ for $10 \mathrm{~min}$, vortexed, and placed on ice for $10 \mathrm{~min}$ before centrifugation at $16,000 \mathrm{~g}$ for $10 \mathrm{~min}$ at $4^{\circ} \mathrm{C}$. Supernatants were transferred to $1.5 \mathrm{~mL}$ DNase/RNase-free tubes (Eppendorf, NY) and analyzed immediately or frozen until analysis.

A minimum of $20 \mu \mathrm{L}$ of samples (plasma, serum, or liver), standards, and QCs was then transferred into a 96-well plate and placed into a preheated thermal cycler (Mastercycler ${ }^{\circledR}$; Eppendorf) at $95^{\circ} \mathrm{C}$ for $10 \mathrm{~min}$ to allow the duplexes to denature and facilitate the annealing of the stem-loop primer to the antisense strand of the siRNA during the reverse transcription (RT) reaction.

\section{Stem-loop RT-qPCR}

To detect siRNAs using a TaqMan ${ }^{\circledR}$-based approach, we used a similar strategy to the one described by Chen et al. [18] for microRNA detection. The approach is illustrated in Fig. 2. In brief, the RT step occurs in the presence of a stemloop RT primer that is complementary to the last 6-10 bases of the $3^{\prime}$ end of the antisense strand of the target siRNA. The stem-loop primer contains an additional universal sequence at the $5^{\prime}$ end that facilitates a TaqMan-based detection strategy in the subsequent qPCR step. As in the case of microRNA, the forward primer for qPCR is sequencespecific for the target siRNA. For sequence compositions that yield a low predicted melting temperature $\left(\mathrm{T}_{\mathrm{m}}\right)$, the forward primer is designed as a tailed primer to help increase $\mathrm{T}_{\mathrm{m}}$.

All samples, standards, and QCs were analyzed in duplicate. Each assay plate included a standard curve containing at least
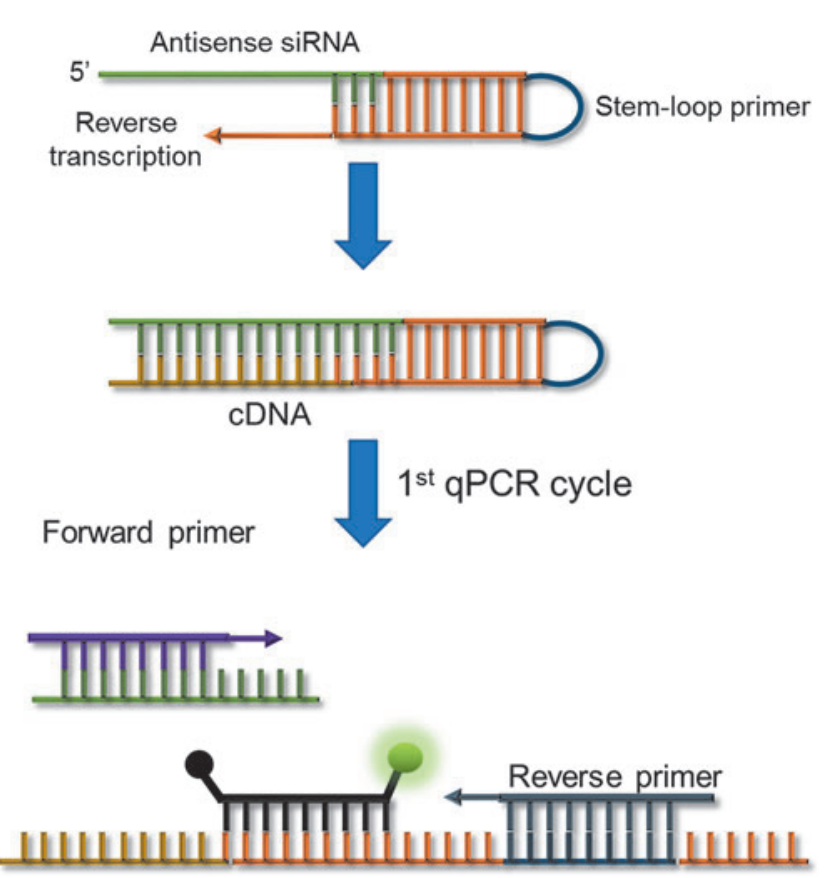

FIG. 2. Schematic representation of a stem-loop reverse transcription followed by a qPCR approach to quantify the antisense strand (full-length or active drug component). RTqPCR, reverse transcription quantitative polymerase chain reaction. 
A

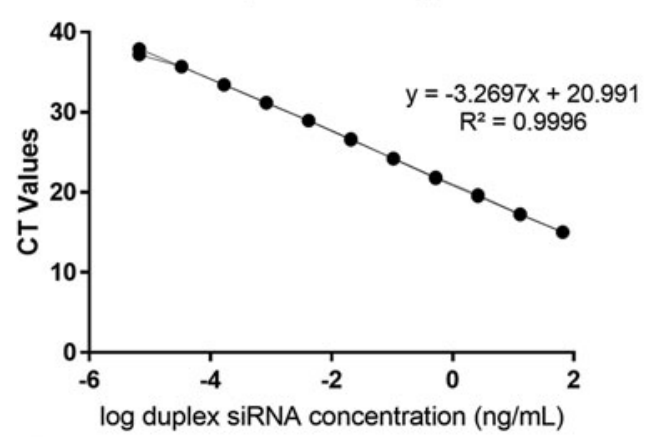

\begin{tabular}{ccccccc}
\hline $\begin{array}{c}\text { Log duplex } \\
\text { siRNA } \\
\text { concentration } \\
\text { (ng/mL) }\end{array}$ & $\begin{array}{c}\text { siRNA } \\
\text { concentration } \\
\text { (ng/mL) }\end{array}$ & $\begin{array}{c}\text { Dilution } \\
\text { factor }\end{array}$ & $\begin{array}{c}\text { CT value } \\
\text { replicate 1 }\end{array}$ & $\begin{array}{c}\text { CT value } \\
\text { replicate 2 }\end{array}$ & $\begin{array}{c}\text { Standard } \\
\text { deviation }\end{array}$ & $\begin{array}{c}\text { Mean } \\
\text { CT } \\
\text { value }\end{array}$ \\
\hline 1.81 & 65.19 & & 15.0 & 15.0 & 0.01 & 15.0 \\
1.12 & 13.04 & & 17.2 & 17.3 & 0.02 & 17.2 \\
0.42 & 2.61 & & 19.5 & 19.7 & 0.07 & 19.6 \\
-0.28 & 0.52 & & 21.7 & 21.9 & 0.06 & 21.8 \\
-0.98 & 0.10 & 1.5 & 24.2 & 24.2 & 0.03 & 24.2 \\
-1.68 & 0.02 & & 26.7 & 26.6 & 0.06 & 26.6 \\
-2.38 & 0.004 & & 31.2 & 31.1 & 0.02 & 29.0 \\
-3.08 & 0.000834 & & 33.5 & 33.4 & 0.03 & 31.2 \\
-3.78 & 0.000167 & & 35.7 & 35.7 & 0.01 & 33.4 \\
-4.48 & 0.000033 & & 37.9 & 37.2 & 0.37 & 37.7 \\
-5.18 & 0.000007 & & 15.0 & 15.0 & 0.01 & 15.0 \\
-5.87 & 81 & & 18.0 & 17.9 & 0.06 & 17.9 \\
1.91 & 8.1 & & 21.1 & 21.1 & 0.03 & 21.1 \\
0.91 & 0.81 & $1: 10$ & 24.5 & 24.5 & 0.00 & 24.5 \\
-0.09 & 0.081 & & 28.0 & 28.0 & 0.02 & 28.0 \\
-1.09 & 0.008 & & 31.2 & 31.1 & 0.06 & 31.2 \\
-2.09 & 0.00081 & & & & & \\
\hline
\end{tabular}

B

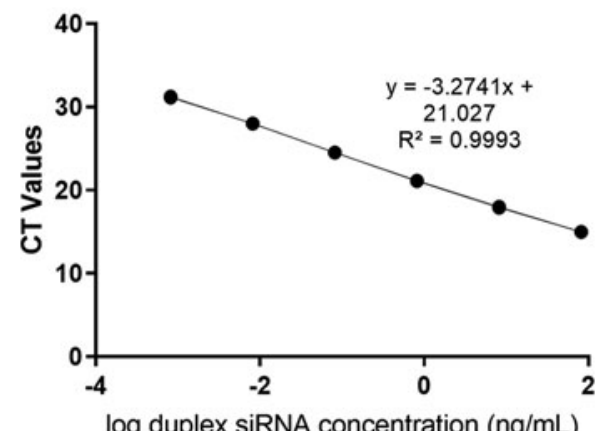

FIG. 3. Analytical sensitivity of stem-loop RT-qPCR on siRNA-1 in serum (A) 1:5 dilution series (B) 1:10 dilution series. six points unless otherwise noted, as well as five levels of QC ranging from 50 to $0.005 \mathrm{nM}$. An assay run was considered acceptable when amplification efficiency fell within 90\%$110 \%$ as calculated from the slope of the log-linear portion of the standard curve, the coefficient of correlation for the stan-

A

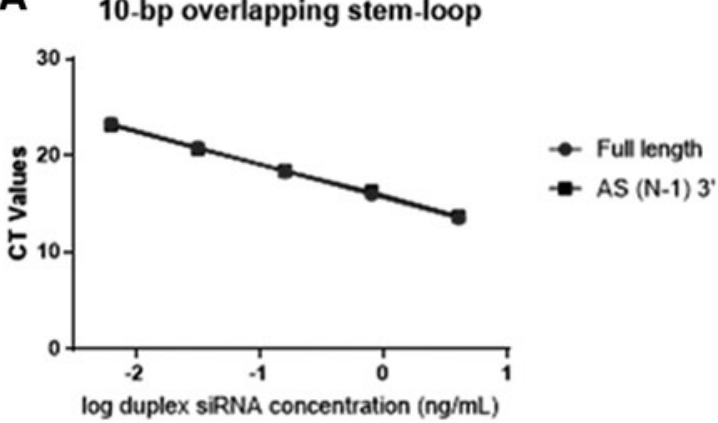

\begin{tabular}{|c|c|c|}
\hline \multirow{2}{*}{$\begin{array}{c}\text { Log duplex siRNA } \\
\text { concentration (ng/mL) }\end{array}$} & \multicolumn{2}{|c|}{ Average CT ( $\pm S D)$} \\
\hline & Full-length & AS (N-1) 3' \\
\hline 0.60206 & $13.59 \pm 0.07$ & $13.83 \pm 0.17$ \\
\hline-0.09691 & $15.78 \pm 0.15$ & $15.98 \pm 0.27$ \\
\hline-0.79588 & $18.18 \pm 0.13$ & $18.36 \pm 0.04$ \\
\hline-1.49485 & $20.75 \pm 0.09$ & $20.58 \pm 0.08$ \\
\hline-2.19382 & $23.34 \pm 0.02$ & $23.02 \pm 0.12$ \\
\hline
\end{tabular}

dard curve was $>0.99$, and the difference between the nominal and observed concentration for each QC level was $\leq 25 \%$.

RT conditions used in this study were the same as previously described by Landesman et al. [19]. The TaqMan MicroRNA Reverse Transcription Kit (Applied Biosystems ${ }^{\circledR}$; ThermoFisher
B 6-bp overlapplng stem-loop

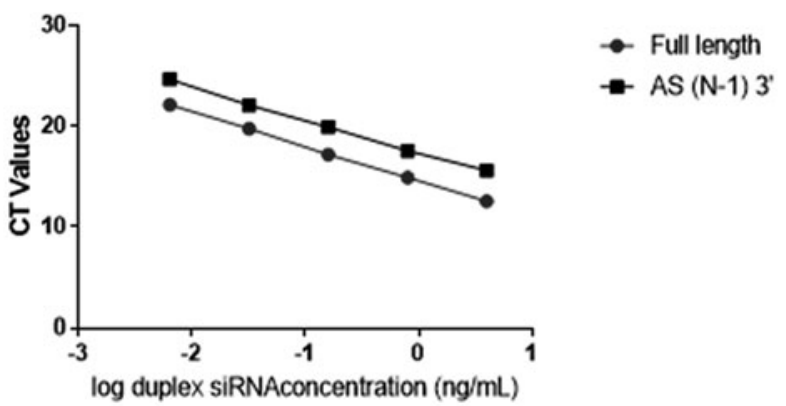

\begin{tabular}{ccc}
\hline Log duplex siRNA & \multicolumn{2}{c}{ Average CT ( \pm SD) } \\
\cline { 2 - 3 } concentration $(\mathbf{n g} / \mathrm{mL})$ & Full-length & AS (N-1) 3' \\
\hline 0.60206 & $12.59 \pm 0.02$ & $15.56 \pm 0.04$ \\
-0.09691 & $14.93 \pm 0.01$ & $17.53 \pm 0.03$ \\
-0.79588 & $17.14 \pm 0.04$ & $19.89 \pm 0.02$ \\
-1.49485 & $19.79 \pm 0.02$ & $22.08 \pm 0.00$ \\
-2.19382 & $22.24 \pm 0.12$ & $24.68 \pm 0.03$ \\
\hline
\end{tabular}

FIG. 4. Stem-loop RT strategy using either a $10 \mathrm{bp}$ (A) or a 6 bp overlapping (B) stem-loop primer. Standard curve comparison between both primer strategies for the detection of full-length siRNA-2 and AS(N-1) $3^{\prime}$ siRNA. bp, base pair. 


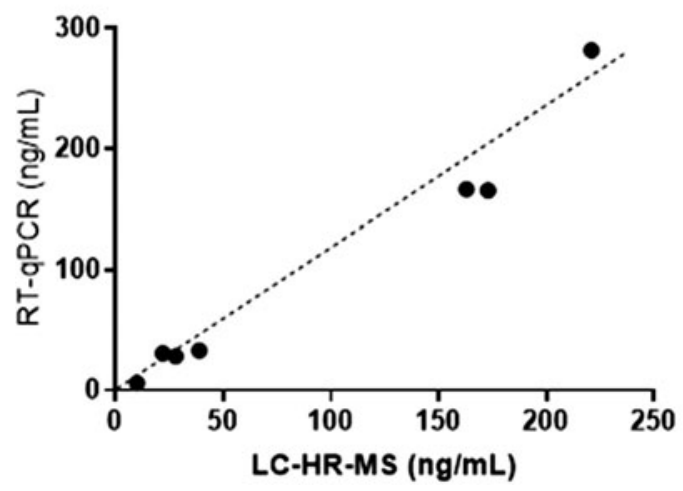

\begin{tabular}{|l|l|}
\hline Pearson $r$ & \\
\hline$r$ & 0.9823 \\
\hline 95\% confidence interval & 0.8809 to 0.9975 \\
\hline$R$ squared & 0.965 \\
\hline & \\
\hline$P$ value & \\
\hline$P$ (two-tailed) & $<0.0001$ \\
\hline$P$ value summary & $\mathbf{1 + *}$ \\
\hline Significant? (alpha $=0.05)$ & Yes \\
\hline & \\
\hline Number of XYPairs & 7 \\
\hline
\end{tabular}

FIG. 5. Pearson correlation between siRNA-1 concentrations as measured by either mass spectrometry (LCHR-MS) or RT-qPCR from seven different human serum samples after single dose at either 1 or $6 \mathrm{mg} / \mathrm{kg}$ for the first $24 \mathrm{~h}$. LC-HR-MS, liquid chromatography-high resolution mass spectrometry.

A SIRNA-4 assay repeatability

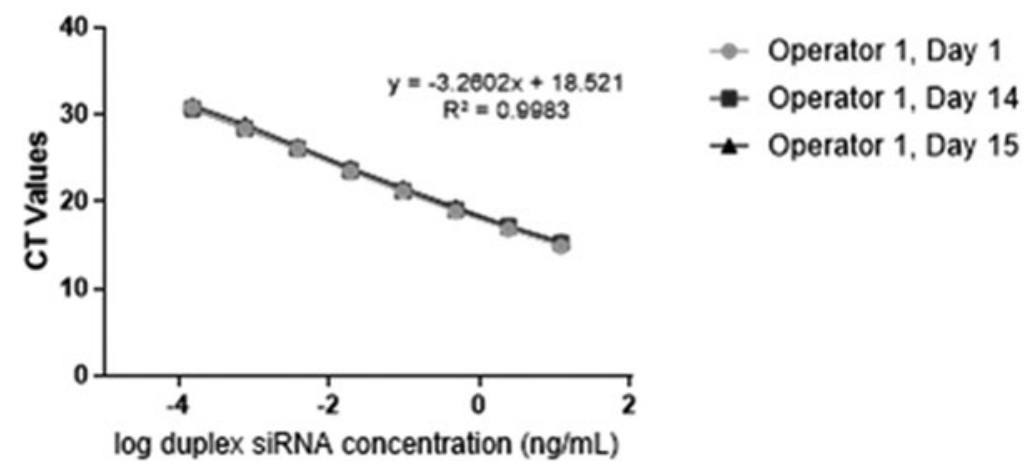

\begin{tabular}{ccccc}
\hline siRNA concentration $\mathbf{n g} / \mathbf{m L}(\mathbf{l o g})$ & Day 1 & Day 14 & Day 15 & Standard deviation \\
\hline 1.08 & 14.9 & 15.3 & 15.4 & 0.22 \\
0.38 & 16.9 & 17.2 & 17.2 & 0.14 \\
-0.32 & 18.9 & 19.1 & 19.4 & 0.21 \\
-1.02 & 21.2 & 21.2 & 21.6 & 0.19 \\
-1.72 & 23.5 & 23.6 & 23.9 & 0.17 \\
-2.42 & 26.1 & 26.1 & 26.4 & 0.14 \\
-3.12 & 28.4 & 28.4 & 28.9 & 0.24 \\
-3.82 & 30.8 & 30.7 & 31.1 & 0.17 \\
\hline
\end{tabular}

B SIRNA-4 assay reproducibility

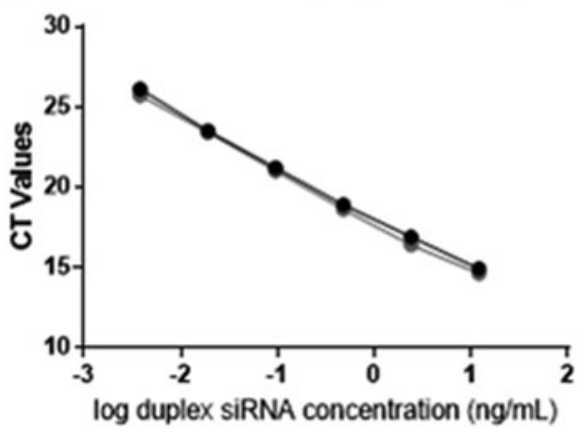

Operator 1

$\rightarrow$ Operator 2

\begin{tabular}{cccc}
\hline siRNA concentration $\mathbf{~ n g / m L ~}(\log )$ & Operator $\mathbf{~}$ & Operator $\mathbf{~}$ & Standard deviation \\
\hline 1.08 & 14.9 & 14.6 & 0.15 \\
0.38 & 16.9 & 16.4 & 0.25 \\
-0.32 & 18.9 & 18.6 & 0.15 \\
-1.02 & 21.2 & 21.0 & 0.10 \\
-1.72 & 23.5 & 23.4 & 0.05 \\
-2.42 & 26.1 & 25.7 & 0.20 \\
\hline
\end{tabular}

FIG. 6. (A) Intra-operator assay performance across multiple days using the same siRNA-4 stock and (B) inter-operator variability the same day. 
Scientific) was used following manufacturer's recommendations. In brief, $5 \mu \mathrm{L}$ of the denatured sample/standard was added to $10 \mu \mathrm{L}$ of the RT reaction mixture containing $10 \times$ buffer, $1 \times$ reverse transcriptase, RNAse inhibitor, stem-loop oligonucleotide, water, and dNTPs. Reactions were then incubated at $16^{\circ} \mathrm{C}$ for $30 \mathrm{~min}$, followed by $42^{\circ} \mathrm{C}$ for $30 \mathrm{~min}$, and a final inactivation step at $85^{\circ} \mathrm{C}$ for $5 \mathrm{~min}$.

The qPCR step was then performed on a ViiA 7 Real-Time PCR System (Applied Biosystems; ThermoFisher Scientific) using a 384-well block and TaqMan ${ }^{\mathrm{TM}}$ Fast Advanced Master Mix (ThermoFisher Scientific) according to the manufacturer's protocols.

Sequence-specific stem-loop, forward and reverse primers (Integrated DNA Technologies, IA), and TaqMan probes (ThermoFisher Scientific) were also designed as previously reported by Chen et al. [18] and Landesman et al. [19]. For the stem-loop primer, the universal sequence (5' GTCGTATCC AGTGCAGGGTCCGAGGTATTCGCACTGGATACGAC 3') reported by Jung et al. [20] was used. Primer Express Software v3.0.1 (ThermoFisher Scientific) was used to predict $\mathrm{T}_{\mathrm{m}}$ values for primers and minor groove binder-containing probes and to evaluate the presence of potential secondary structures.

\section{Measurement of siRNA loaded into RISC}

Measurement of RISC-loaded siRNA (antisense strand) in liver was performed as previously reported by Nair et al. [21] using a combination of immunoprecipitation followed by the stem-loop RT-qPCR procedure described earlier.

Processed (powdered) liver prepared from $50 \mathrm{mg}$ of tissue was resuspended at $100 \mathrm{mg} / \mathrm{mL}$ in prechilled lysis buffer $(50 \mathrm{mM}$ Tris- $\mathrm{HCl}, \mathrm{pH} 7.5,150 \mathrm{mM} \mathrm{NaCl}, 2 \mathrm{mM}$ EDTA, $0.5 \%$ Triton-X 100; chemicals were obtained from ThermoFisher Scientific), supplemented with one tablet of cOmplete $^{\mathrm{TM}}$ Mini, EDTA-free Protease Inhibitor Cocktail (Roche, Sigma-Aldrich) and $1 \mathrm{mM}$ of phenylmethanesulfonyl fluoride (Sigma-Aldrich). Precleared samples (using QAESephadex ${ }^{\circledR}$ A-50 resin; Sigma-Aldrich) were then subjected to Ago2 immunoprecipitation by incubation with anti-mouse Ago2 (Clone No: 2D4; FUJIFILM Wako Pure Chemical Corporation, San Diego, CA) and control normal mouse IgG (sc-2025; Santa Cruz Biotechnology, Inc., Dallas, TX) followed by precipitation with Protein G Dynabeads (Life Technologies, ThermoFisher Scientific). Final lysates of RISC-loaded siRNAs were subsequently quantified by the stem-loop RT-qPCR approach described earlier.

\section{mRNA quantification}

Total mRNA was isolated, and reverse transcribed into cDNA as previously described [15]. qPCR reactions were performed using gene-specific TaqMan assays for each target gene (Mm00443267_m1 for Ttr, and Mm00551119_m1 for Tmprss6; ThermoFisher Scientific).

\section{Data analysis}

Mean, standard deviation, and linear regression were calculated in GraphPad Software V 7.01 G (GraphPad Software, San Diego, CA). The slope of the regression line was calculated using the log-transformed siRNA concentration $(\mathrm{ng} / \mathrm{mL})$ as the independent variable versus the mean cycle threshold (CT) value (number of PCR cycles for the fluorescence signal to cross the threshold) as the dependent variable.

\section{Results}

\section{Reportable range and linearity}

An example of reportable range and linearity for siRNA-1 using the RT-qPCR method is shown in Fig. 3. In this example, the standard curve was generated using 11 standards. The siRNA concentrations ranged over $6 \operatorname{logs}(65.19 \mathrm{ng} / \mathrm{mL}$ to $1.3 \times 10^{-6} \mathrm{ng} / \mathrm{mL}$ ), including the anticipated quantification range. The $\mathrm{qPCR}$ reaction had a calculated efficiency of $102 \%$, based on the slope of -3.27 .

\section{Analytical specificity and accuracy}

Mass spectrometry can discriminate full-length siRNA and metabolites with a resolution of $1 \mathrm{bp}$ in contrast to the stemloop RT-qPCR approach that detects all metabolites plus fulllength as total siRNA without discriminating (Table 1).

The stem-loop primers were designed to have either a 6-bp or a 10-bp overlap to the $3^{\prime}$ end of the antisense strand of siRNA. Assay performance using both types of primer was assessed based on the standard curves generated by RT-qPCR of intact siRNA-2 and siRNA-2 lacking the $3^{\prime}$ terminal nucleotide on the antisense strand, $\mathrm{AS}(\mathrm{N}-1) 3^{\prime}$. As shown in Fig. 4A, RT-qPCR using the 10-bp overlapping stem-loop primer detected full-length siRNA-2 and AS(N-1)3' sequence with the
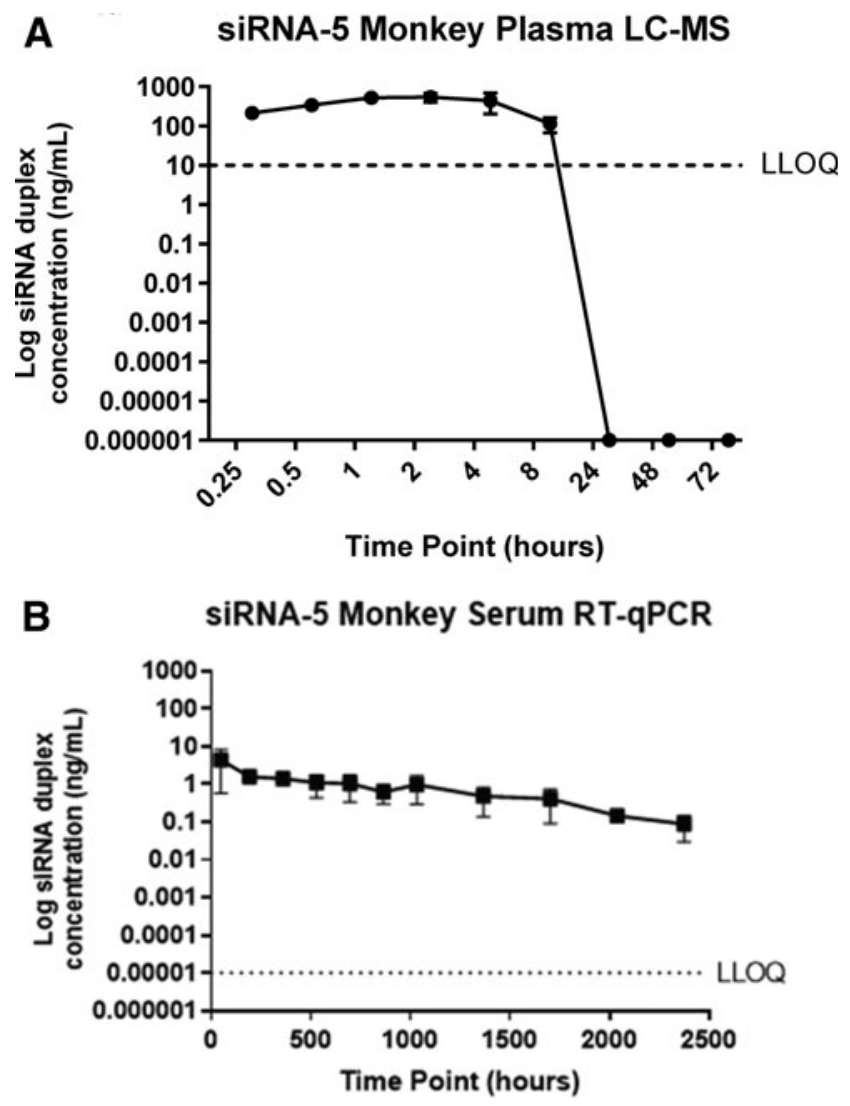

FIG. 7. Mass spectrometry and RT-qPCR plasma and serum siRNA-5 profiles for nonhuman primates after one SC dose at $0.3 \mathrm{mg} / \mathrm{kg}$. Mass spectrometry plasma profiles fall below LLOQ after only $8 \mathrm{~h}$ postdose (A). RT-qPCR assay was able to detect siRNA-5 in serum up to $2,376 \mathrm{~h}$ (99 days) (B). LLOQ, lower limit of quantification; SC, subcutaneous. 
same accuracy. However, assay accuracy for the detection of the $\mathrm{AS}(\mathrm{N}-1) 3^{\prime}$ siRNA was lower when using the 6-bp overlapping stem-loop primer (Fig. 4B). When the 6-bp overlapping stemloop primer was used, the $\mathrm{AS}(\mathrm{N}-1) 3^{\prime}$ siRNA had an average CT delay of 2.88 when compared with the full-length sequence, indicating a reduction in recovery and lowered assay accuracy.

To corroborate that the aforementioned findings were applicable to other siRNA sequences, we performed the assay using the 6-bp overlapping stem-loop primer with a different siRNA sequence (siRNA-3) and its $\mathrm{AS}(\mathrm{N}-1) 3^{\prime}$ form, together with a truncation variant where one nucleotide was removed from the $5^{\prime}$ terminus of the antisense strand, AS(N-1)5' (Supplementary Fig. S1). Results showed that the loss of only one nucleotide on the $3^{\prime}$ end of the antisense strand of the siRNA resulted in a CT delay of 4.7 CT for this particular sequence compared with the full-length antisense strand. For the AS(N-1) $5^{\prime}$ form, CT values were the same as for the full-length sequence.

To measure the closeness between the measured value and the true value we also compared siRNA-1 concentrations measured by stem-loop RT-qPCR versus mass spectrometry in the serum of human healthy volunteers after a single dose at either 1 or $6 \mathrm{mg} / \mathrm{kg}$. Comparison between siRNA concentrations up to $24 \mathrm{~h}$ showed a Pearson correlation of 0.965 between the two methods as calculated by GraphPad Software V 7.01 (Fig. 5).

\section{Precision}

To assess siRNA ADME properties, assays must be precise, robust, and reproducible, yielding the same values from repeat analysis of the same input material across different days and operators.

Figure 6 shows assay repeatability for the same operator (intra-operator) as a function of $\mathrm{CT}$ values across several siRNA-4 input concentrations for a 15-day period. Figure 6B also reflects the assay reproducibility, assessed by two independent operators on the same day using the same analyte, siRNA-4.
A

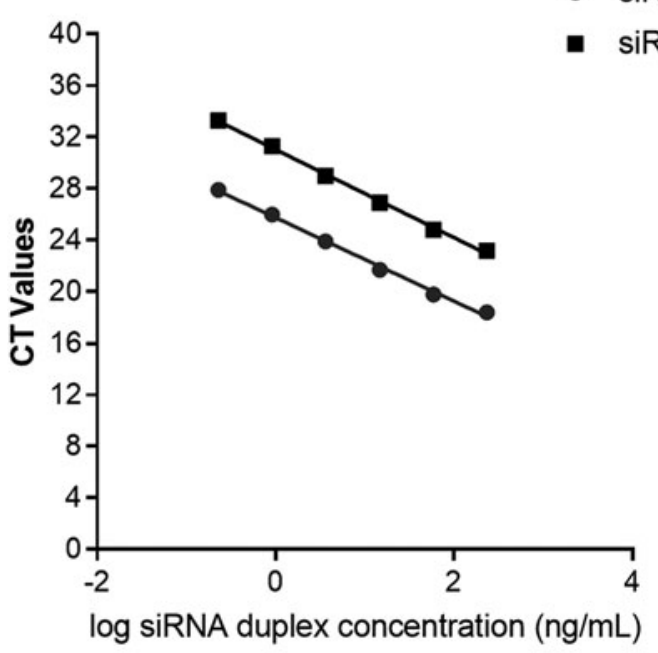

$1 \mathrm{mM} \mathrm{MnCl}{ }_{2}$

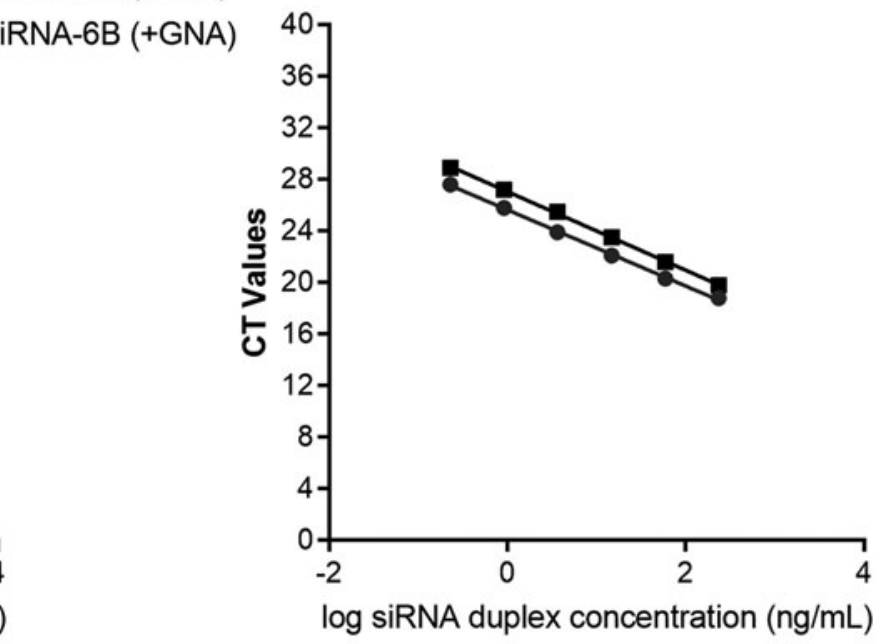

B siRNA concentration by LC-MS and RT-qPCR

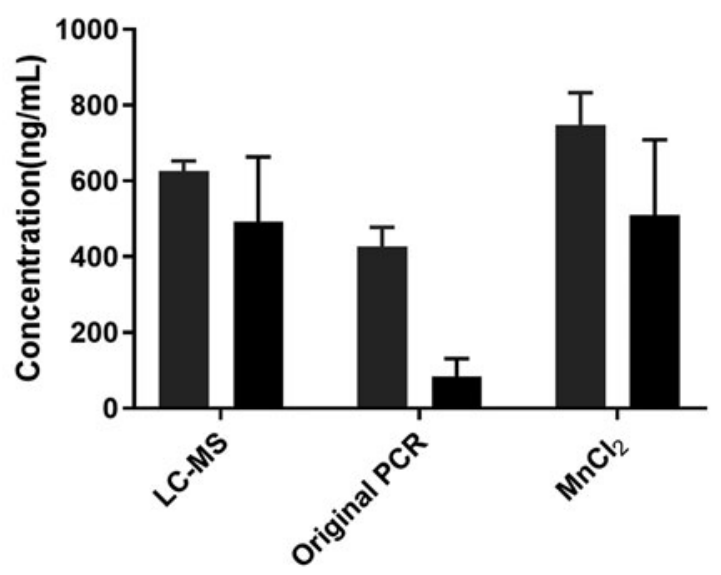

- siRNA-7A (-GNA)

- siRNA-7B (+GNA) 
Sensitive siRNA detection with RT-qPCR for in vivo samples

Figure 7 depicts the plasma and serum profiles for nonhuman primates $(n=3)$ for siRNA-5 after a single subcutaneous dose at $3 \mathrm{mg} / \mathrm{kg}$ measured by mass spectrometry (Fig. 7A) and RT-qPCR (Fig. 7B). siRNA levels in plasma samples collected $>8 \mathrm{~h}$ postdose fall below lower limit of quantification (LLOQ) when analyzed by mass spectrometry (serum has a similar sensitivity, data not shown), whereas siRNA-5 was still detected up to 2,376 h (99 days) using RT-qPCR. For siRNA-5, the LLOQ for the RT-qPCR assay was $>1$ million-fold lower than for mass spectrometry $(6.71 \mathrm{fg} / \mathrm{mL}$ vs. $10 \mathrm{ng} / \mathrm{mL}$ ).

\section{Effect of siRNA modifications on RT-qPCR detection}

Standard curves of siRNA-6 with and without a single GNA modification showed that the incorporation of a single GNA base resulted in a $6 \mathrm{CT}$ shift in the standard curve compared with the same duplex siRNA without the GNA base. In this case, both standard curves still showed qPCR efficiencies between $90 \%$ and $110 \%$ (Fig. 8A). This is not surprising since previous studies [22] have shown that the presence of this three-carbon acyclic backbone base is an unnatural substrate for reverse transcriptases and that only the addition of manganese (II) ions improved GNAdependent DNA synthesis. They demonstrated that the incorporation of $1 \mathrm{mM}$ manganese chloride $\left(\mathrm{MnCl}_{2}\right)$ into the RT step led to increased full-length cDNA product.

For the siRNA-6 sequence, we also demonstrated that the incorporation of $1 \mathrm{mM} \mathrm{MnCl}_{2}$ during the cDNA synthesis step helped decrease the CT shift previously observed between the non-GNA (parent) and GNA-containing duplexes.

Next, to assess whether this strategy could be applied to samples from animals treated with a different siRNA duplex, we measured plasma concentrations for siRNA-7 from nonhuman primates $2 \mathrm{~h}$ after dosing at $3 \mathrm{mg} / \mathrm{kg}$. As shown in Fig. $8 \mathrm{~B}$, siRNA concentrations using $\mathrm{MnCl}_{2}$ in the RT-qPCR reaction showed similar siRNA concentrations $(\mathrm{ng} / \mathrm{mL})$ for the GNA and non-GNA, compared with the mass spectrometry data (GNA does not influence the performance of this method, Supplementary Fig. S2). As shown in Fig. 8B, samples from animals treated with the GNA version of siRNA-7 were inaccurately quantified with the previous RTqPCR strategy.

\section{Correlation of siRNA levels with RISC-loaded drug and pharmacodynamic profiles}

Very high assay sensitivity is crucial when evaluating the active form of siRNA therapeutics (ie, RISC-loaded drug). For this, the RT-qPCR method presented here can accurately measure siRNAs loaded into RISC, which are typically $<1 \mathrm{ng} / \mathrm{g}$ (ng siRNA per gram liver tissue) as shown in Fig. 9. In these experiments, two siRNAs targeting two different targets, transmembrane serine protease 6 (Tmprss6) and mouse transthyretin (Ttr) were evaluated in animals receiving single SC doses of 2.5 and $0.75 \mathrm{mg} / \mathrm{kg}$. The TMP siRNA was ESC (Enhanced Stabilization Chemistry) and the TTR siRNA was Advanced
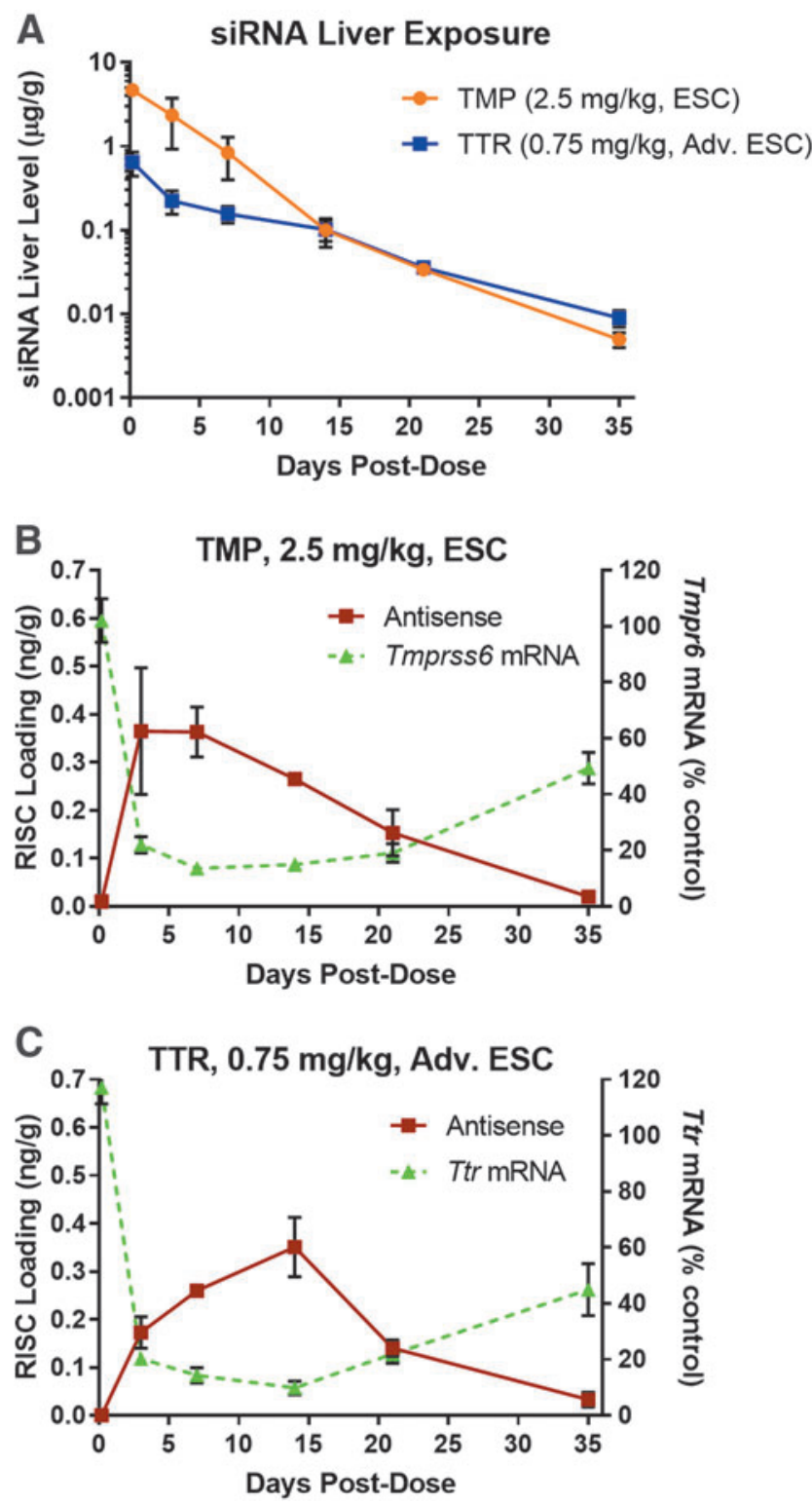

FIG. 9. Utility of RT-qPCR for sensitive quantification of RISC-loaded drug and correlation with pharmacodynamic profiles from target liver tissues. (A) Antisense liver exposure was quantified using RT-qPCR against siRNAs targeting Tmprss6 mRNA (TMP, $2.5 \mathrm{mg} / \mathrm{kg}$ ) and Ttr mRNA (TTR, $0.75 \mathrm{mg} / \mathrm{kg}$ ). The siRNAs were either ESC (TMP) or Advanced ESC (TTR), which is a more stable design. Concentration of siRNA is presented as $\mu \mathrm{g}$ siRNA per gram of liver $(\mu \mathrm{g} / \mathrm{g})$. (B) Knockdown of Tmprss6 mRNA (green line) by an ESC-design siRNA delivered by a single subcutaneous dose $(2.5 \mathrm{mg} / \mathrm{kg})$. RISC-loaded antisense siRNA was quantified by RT-qPCR (red). Units are in ng antisense siRNA per gram of liver (ng/g). (C) Knockdown of Ttr mRNA (green line) by an Advanced ESC-design siRNA delivered by a single subcutaneous dose $(0.75 \mathrm{mg} / \mathrm{kg})$. RISC-loaded antisense siRNA was quantified by RT-qPCR (red). Units are in ng antisense siRNA per gram of liver (ng/g). ESC, Enhanced Stabilization Chemistry; mRNA, messenger RNA; RISC, RNA-induced silencing complex. 
ESC, a more metabolically stable design. Total liver exposure was measured for the antisense strand of both siRNAs (Fig. 9A). The TMP siRNA had higher initial levels due to the higher dose but was eliminated faster than the TTR siRNA. RISC-loaded antisense siRNA was quantified by RT-qPCR and showed a direct correlation with target mRNA knockdown.

\section{Discussion}

To assess ADME properties of various siRNAs at the preclinical stage, it is important to quantify them accurately from various matrices without having to use time-consuming or costly isolation methods with variable efficiencies. Stemloop RT-qPCR is a very high-throughput method and assay efficiency is not impacted by the presence of crude biological matrices with variable nonspecific binding properties [19,23].

In addition, to understand the mechanism of action of different siRNA sequences and chemical modifications at the discovery stage, it is necessary to have a sensitive method that can measure how much duplex is loaded into RISC. For this, hybridization enzyme-linked immunosorbent assay (ELISA) [24] or RT-qPCR could provide the necessary assay sensitivity $(<1 \mathrm{ng} / \mathrm{g})$ with medium- to high-throughput capabilities, respectively. However, one of the reported caveats of hybridization ELISA is its lower specificity compared with mass spectrometry, which has been addressed with novel approaches such as dual ligation hybridization $[9,13]$. Although hybridization ELISA provides a good alternative to RT-qPCR, it still requires seven primer/template/probe oligonucleotides and three different enzymes, a complex approach that may pose some challenges in routine bioanalysis, especially when dealing with many matrices and siRNA sequences.

Understanding RISC pharmacokinetics (PK) in preclinical species can assist in the development of quantitative models that predict reduction of target mRNA in humans. These predictions can help determine appropriate dose levels and dose frequency in future clinical trials. The RT-qPCR method is critical to these evaluations due to its exquisite sensitivity for the low drug levels present in RISC.

In addition, to quantify siRNAs with the same sequence but differing chemical modifications, RT-qPCR uses the same set of primers/probes (if equivalent RT and amplification efficiencies are previously established), whereas mass spectrometry requires specific method development for each unique siRNA.

Despite the advantages of RT-qPCR for siRNA analysis, it is still critical to assess intact siRNA (full-length) and its metabolites to design more stable and potent molecules. For this, mass spectrometry is to date the only bioanalytical method capable of achieving this level of specificity. Thus, it is still best to choose downstream bioanalytical methods based on best fit for purpose contingent on throughput, sensitivity, and specificity needs.

In summary, RT-qPCR provides high throughput and high sensitivity with tolerance for a variety of biological matrices. In addition, this method expedites the evaluation of any set of siRNAs having the same sequence but with distinct chemical modifications by utilizing a single set of primers for all analyses.

\section{Acknowledgment}

We thank Sarah Bond for careful review of the article and for providing comments and insights.

\section{Author Disclosure Statement}

All authors were employees of Alnylam Pharmaceuticals, Inc. at the time of this study.

\section{Funding Information}

Internal funding for this work was provided by Alnylam Pharmaceuticals. No funding was received from outside institutions or funding agencies.

\section{Supplementary Material}

Supplementary Figure S1

Supplementary Figure S2

\section{References}

1. Adams D, A Gonzalez-Duarte, WD O'Riordan, CC Yang, M Ueda, AV Kristen, I Tournev, H Schmidt, T Coelho, et al. (2018). Patisiran, an RNAi therapeutic, for hereditary transthyretin amyloidosis. N Engl J Med 379:11-21.

2. U.S. Food \& Drug Administration Drugs@FDA. (2019). FDA Approved Drug Products. New Drug Application (NDA): 212194 Givlaari. Approval for Givlaari.

3. Carthew RW and EJ Sontheimer. (2009). Origins and mechanisms of miRNAs and siRNAs. Cell 136:642-655.

4. Alemán LM, J Doench and PA Sharp. (2007). Comparison of siRNA-induced off-target RNA and protein effects. RNA 13:385-395.

5. Martinez J, A Patkaniowska, H Urlaub, R Luhrmann and T Tuschl. (2002). Single-stranded antisense siRNAs guide target RNA cleavage in RNAi. Cell 110:563-574.

6. Janas MM, Y Jiang, RG Duncan, AN Hayes, J Liu, PV Kasperkovitz, ME Placke and SA Barros. (2016). Exposure to siRNA-GalNAc conjugates in systems of the standard test battery for genotoxicity. Nucleic Acid Ther 26:363371.

7. Nair JK, JL Willoughby, A Chan, K Charisse, MR Alam, Q Wang, M Hoekstra, P Kandasamy, AV Kel'in, et al. (2014). Multivalent $\mathrm{N}$-acetylgalactosamine-conjugated siRNA localizes in hepatocytes and elicits robust RNAi-mediated gene silencing. J Am Chem Soc 136:16958-16961.

8. Springer AD and SF Dowdy. (2018). GalNAc-siRNA conjugates: leading the way for delivery of RNAi therapeutics. Nucleic Acid Ther 28:109-118.

9. Wang L. (2011). Oligonucleotide bioanalysis: sensitivity versus specificity. Bioanalysis 3:1299-1303.

10. Basiri B and MG Bartlett. (2014). LC-MS of oligonucleotides: applications in biomedical research. Bioanalysis 6: 1525-1542.

11. Kaczmarkiewicz A, L Nuckowski, S Studzinska and B. Buszewski. (2019). Analysis of antisense oligonucleotides and their metabolites with the use of ion pair reversed-phase liquid chromatography coupled with mass spectrometry. Crit Rev Anal Chem 49:256-270.

12. Lin ZJ, W Li and G Dai. (2007). Application of LC-MS for quantitative analysis and metabolite identification of therapeutic oligonucleotides. J Pharm Biomed Anal 44:330 341. 
13. Tremblay GA and PR Oldfield. (2009). Bioanalysis of siRNA and oligonucleotide therapeutics in biological fluids and tissues. Bioanalysis 1:595-609.

14. van Dongen WD and WM Niessen. (2011). Bioanalytical LC-MS of therapeutic oligonucleotides. Bioanalysis 3: 541-564.

15. Foster DJ, CR Brown, S Shaikh, C Trapp, MK Schlegel, K Qian, A Sehgal, KG Rajeev, V Jadhav, et al. (2018). Advanced siRNA designs further improve in vivo performance of GalNAc-siRNA conjugates. Mol Ther 26: 708-717.

16. Janas MM, MK Schlegel, CE Harbison, VO Yilmaz, Y Jiang, R Parmar, I Zlatev, A Castoreno, $\mathrm{H} \mathrm{Xu}$, et al. (2018). Selection of GalNAc-conjugated siRNAs with limited off-target-driven rat hepatotoxicity. Nat Commun 9:723-732.

17. Bustin SA, V Benes, JA Garson, J Hellemans, J Huggett, M Kubista, R Mueller, T Nolan, MW Pfaffl, et al. (2009). The MIQE guidelines: minimum information for publication of quantitative real-time PCR experiments. Clin Chem 55: 611-622.

18. Chen C, DA Ridzon, AJ Broomer, Z Zhou, DH Lee, JT Nguyen, M Barbisin, NL Xu, VR Mahuvakar, et al. (2005). Real-time quantification of microRNAs by stem-loop RTPCR. Nucleic Acids Res 33:e179.

19. Landesman Y, N Svrzikapa, A Cognetta III, X Zhang, BR Bettencourt, S Kuchimanchi, K Dufault, S Shaikh, M Gioia, et al. (2010). In vivo quantification of formulated and chemically modified small interfering RNA by heating-in-Triton quantitative reverse transcription polymerase chain reaction (HIT qRT-PCR). Silence 1: 16-29.
20. Jung U, X Jiang, SH Kaufmann and V Patzel. (2013). A universal TaqMan-based RT-PCR protocol for cost-efficient detection of small noncoding RNA. RNA 19:1864-1873.

21. Nair JK, H Attarwala, A Sehgal, Q Wang, K Aluri, X Zhang, M Gao, J Liu, R Indrakanti, et al. (2017). Impact of enhanced metabolic stability on pharmacokinetics and pharmacodynamics of GalNAc-siRNA conjugates. Nucleic Acids Res 45:10969-10977.

22. Tsai C-H, J Chen and JW Szostak. (2007). Enzymatic synthesis of DNA on glycerol nucleic acid templates without stable duplex formation between product and template. Proc Natl Acad Sci U S A 104:14598-14603.

23. Seitzer J, H Zhang, M Koser, Y Pei and M Abrams. (2011). Effect of biological matrix and sample preparation on qPCR quantitation of siRNA drugs in animal tissues. J Pharmacol Toxicol Methods 63:168-173.

24. Thayer MB, JM Lade, D Doherty, F Xie, B Basiri, OS Barnaby, NS Bala and BM Rock. (2019). Application of locked nucleic acid oligonucleotides for siRNA preclinical bioanalytics. Sci Rep 9:3566-3574.

Address correspondence to: Elena Castellanos-Rizaldos, PhD Alnylam Pharmaceuticals, Inc. 675 West Kendall Street Cambridge, MA 02142 USA

E-mail: ecrizaldos@alnylam.com

Received for publication December 19, 2019; accepted after revision February 19, 2020; published online March 23, 2020. 